\title{
Dynamical Friction in Barred Galaxies
}

\author{
Victor P. Debattista and J. A. Sellwood \\ Rutgers University, PO Box 849, Piscataway, NJ 08855
}

\section{Introduction}

Weinberg's (1985) perturbative calculation of the dynamical friction by halos on bars predicts that bars will be slowed down very quickly. Previous numerical experiments (e.g. Hernquist \& Weinberg 1992) have obtained results that are in agreement with Weinberg's calculation.

Observations, however, suggest that most bars are rotating rapidly. (In this context, a bar is slow if the major axis Lagrange point is far outside the bar, while a fast bar ends at, or near, this Lagrange point.) Neither Weinberg's calculation nor the numerical experiments have, to the present date, been fully self-consistent, so possibly important effects such as resonance capture and the back reaction on the bar were not included.

\section{The Simulation}

We have therefore run a series of wholly self-consistent, three dimensional simulations of disk galaxies with live disk and halo components. Self-consistency was obtained by iteratively integrating the halo distribution function in the presence of the disk and halo potential, then solving for the new potential. The halo distribution functions had a polytropic form: $f(\varepsilon) \propto \varepsilon^{\frac{3}{2}}$ with an energy cut-off so that all initial particles are bound on the grid.

These models were bar unstable, giving us the self-consistent bars our experiments needed. We report here the results of one of these simulations. The canonical simulation was run on a cartesian grid of size $129^{3}$. A Kuz'min-Toomre disk with length scale $d=5$ mesh spaces and truncated at $4 d$ was used. The vertical scale height was $0.4 d$. The disk was initially very cool with Toomre's $Q$ set to 0.05 . The simulation had $3 \times 10^{5}$ equal mass particles, with $30 \%$ of the total mass in the disk. In the initial disk, one rotation at the velocity turnover takes a time of 41.1 (the unit of time is $\sqrt{\frac{d^{3}}{G M}}$, where $M$ is the total mass in the simulation and $G$ is the universal gravitational constant). The disk formed a bar by $t=150$. The simulation ended at $t=2000$.

\subsection{Angular Momentum Transfer}

Figure 1a shows the evolution of angular momentum in the different components of the simulation; the halo had little angular momentum initially. By $t=2000$, the disk had lost about half of its angular momentum to the halo (the small difference between total initial and total final angular momentum accounted for by particle loss from the grid). 

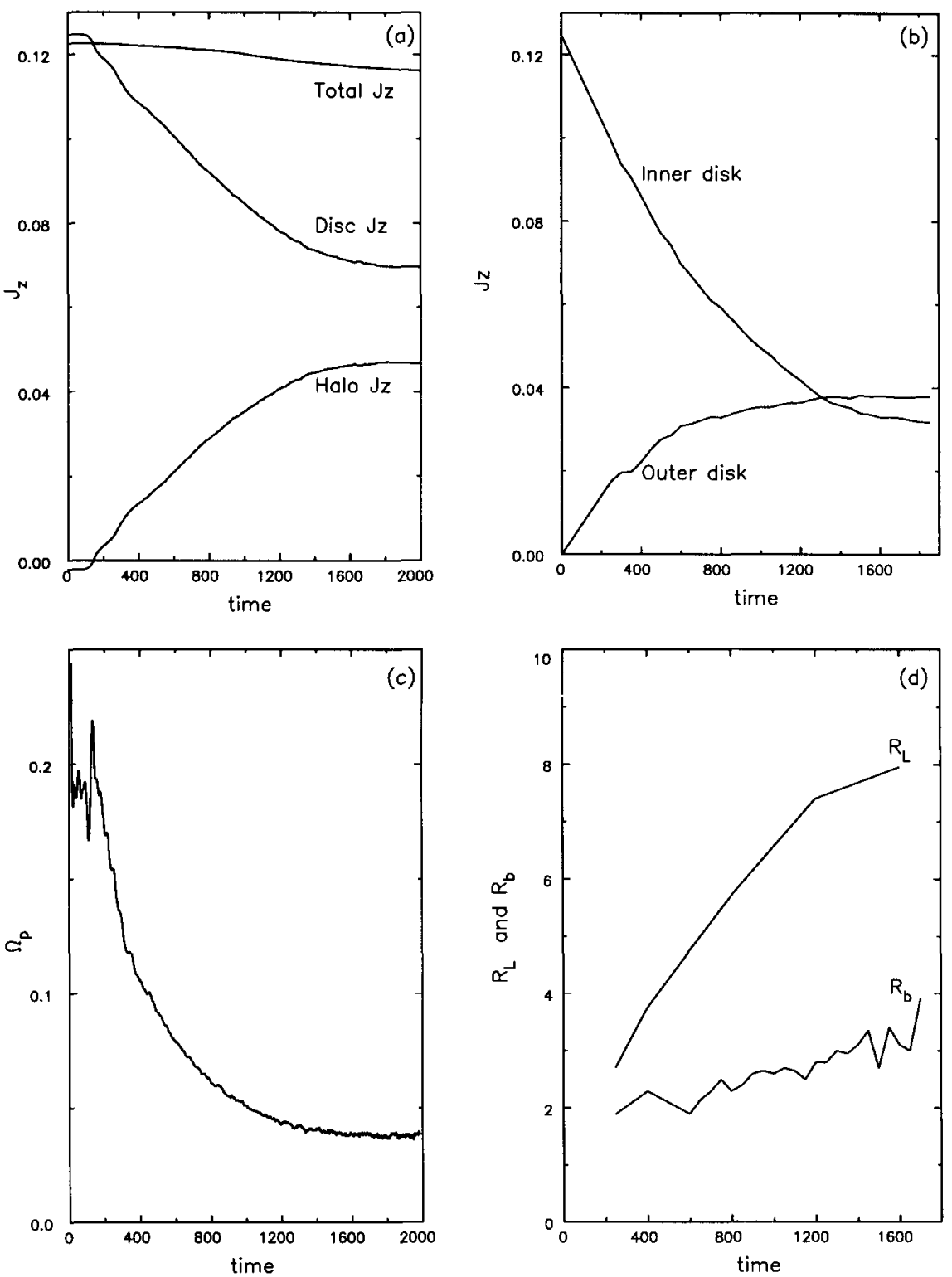

Figure 1. Evolution of physical quantities in the described model. 
Furthermore, one can see from Figure 1b that the angular momentum remaining in the disk is transmitted outwards. Roughly half of the angular momentum from the initial inner disk ( $R \leq 4 d$ where the bar resides) ends up at the outer disk $(R \geq 4 d)$.

\subsection{Evolution of Bar Pattern Speed}

Figure $1 c$ presents the bar pattern speed, $\Omega_{b}(t)$. The initial loss of angular momentum by the bar leads to a very rapid drop in $\Omega_{b}(t)$. An estimate of $T_{\frac{1}{2}}$, the time for $\Omega_{b}$ to drop to one-half the initial value $\Omega_{b}(150)$, is $T_{\frac{1}{2}}=6.7 T_{0}$, where $T_{0}=\frac{2 \pi}{\Omega_{b}(\mathbf{1 5 0 )}}$. This fast decay is of the order of Weinberg's prediction, but eventually, $\Omega_{b}$ asymptotes to a non-zero value, which has not been predicted in the past. This underscores the importance of doing this work self-consistently.

Between $t=200$ and $t=1800$ the angular momentum of the inner disk fell by $\sim 70 \%$. In the same time interval, $\Omega_{b}$ drops by $\sim 77 \%$. Thus the bar behaves as a quasi-solid object, with a positive, approximately constant (with a variation $\sim \pm 20 \%$ ) effective moment of inertia.

It is worth noting that we have managed to obtain a long-lived, slowly rotating bar as the end product of our simulation. The bar has not dissolved into a lens-like structure as Kormendy (1979) has speculated. Thus slow bars seem to be stable objects.

\subsection{Bar Length and Co-rotation}

Finally, Figure 1d shows $R_{b}$, the bar half-length, and $R_{L}$, the distance of L1 (the Lagrange point along the bar major axis) from the center, which is often loosely referred to as co-rotation. It is clear from this figure that, except for when the bar first forms, the bar ends well inside co-rotation. Since our initial disk was truncated at $R=4 d$, we cannot say whether the bar may have been able to grow further if more disk material at a larger radius had been initially available, in which case $R_{b}$ may have been able to keep up with $R_{L}$. (Note however that $R_{b}$ reaches the outer limit of the inner disk long after $R_{L}$ has swept through this region.)

\section{Conclusion}

The results of other simulations (including more realistic warm disks) are in agreement with the results outlined here. Future simulations will test whether it is possible for the bar length to keep growing at a pace to keep up with the outward motion of the Lagrange point. Failing that, there seems to be a real discrepancy between our theoretical understanding of the interaction of bars with their environment and observations.

\section{References}

Hernquist, L. \& Weinberg, M. 1992 ApJ, 400, 80

Kormendy, J. 1979, ApJ, 227, 714

Weinberg, M. 1985, MNRAS, 213, 451 